\title{
VI.1 Sampling alternativer Online-Nachrichtenmedien im DACH-Raum
}

Um Aussagen über den Stellenwert alternativer Nachrichtenmedien in Deutschland, Österreich und der deutschsprachigen Schweiz treffen zu können, muss zunächst das Feld empirisch aufgearbeitet werden. Während eine Auflistung etablierter, professioneller Nachrichtenmedien aufgrund einschlägiger Quellen wie beispielsweise länderspezifischer Auflagenlisten um ein Vielfaches einfacher ist, musste für das Sampling alternativer Newssites ein noch stärkerer induktiver Ansatz gewählt werden. Als problematisch erwies sich an diesem ersten empirischen Schritt zudem die bis dahin wenig eindeutige Definition von Alternativmedien, wie im Forschungsstand bereits erörtert wurde. Um das Feld abzugrenzen und dennoch nicht zu sehr einzuschränken, wurden vorab bestimmte Kriterien festgelegt, wonach, dem klassischen Medienbegriff folgend (Saxer, 1999), Kommunikationskanäle untersucht wurden, die auf Dauer ausgerichtet sind, regelmässig aktuelle Nachrichten verbreiten und eine gewisse Form von Organisation (im Gegensatz zur Verbreitung privater Nachrichten) widerspiegeln. Erstens musste es sich um eine Online-Nachrichtenwebsite aus Deutschland, Österreich oder der deutschsprachigen Schweiz handeln. Die Websites waren in weiterer Folge als Ausgangspunkt zu betrachten, von dem aus auf Accounts auf diversen Social-Media-Plattformen wie Facebook, Twitter und YouTube verwiesen wurde. Deswegen wurden in einem ersten empirischen Schritt Websites gesampelt und erst im Anschluss wurde deren Präsenz auf Digitalplattformen miterhoben, und nicht umgekehrt. ${ }^{1}$ Schnell wurde offensichtlich, dass die Akteur_innen tendenziell stärker via eigener Website vertreten sind, als auf den Social-Media-Plattformen (z. B. auf dem Netzwerk Twitter, das im Rahmen der Netzwerkanalyse untersucht wurde). Vom Sample ausgeschlossen wurden sogenannte «geheime», also nicht öffentliche Gruppen auf z. B. Facebook, die gleichzeitig auch bewusst nicht Teil der Analyse sein sollen, zumal der Fokus dieses Projekts auf öffentlicher und nicht privater respektive geschlossener Kommunikation liegt. Geschlossene Gruppen würden nicht unter diese Definition fallen, da ein Zugang nicht für jede_n möglich ist. Als weiteres Kriterium sollten auf der Website regelmässig aktuelle Nachrichten verbreitet werden, in mindestens zweiwöchentlichen Abständen. Die Newssite sollte sich ferner vom «Mainstream», also der hegemonialen Öffentlichkeit aus Politik und Medien, abgrenzen, wobei sich diese Abgrenzung auf das Selbstverständnis, also die subjektive Sicht der Medien, bezieht - wie es auch auf der Website kommuniziert wird. Auch in Anbetracht dieses Kriteriums eignen sich Websites als Untersuchungsmaterial, da diese häufig auf eigens dafür erstellten Subseiten ihr

1 Ebenso wurden für die Netzwerkanalyse in Schritt 3 die URLs der professionellen Nachrichtenmedien («Mainstreammedien») erhoben, um eine Vergleichbarkeit der Samples zu gewährleisten. 
Selbstverständnis publizieren (beispielsweise unter dem Titel «Über uns»). Vom Sample ausgeschlossen wurden Blogs von Einzelpersonen, in denen ausschliesslich subjektive Meinungen veröffentlicht werden und der Fokus nicht auf der Verbreitung von Nachrichten liegt.

Mithilfe dieser Kriterien wurde mittels Schneeballverfahren eine möglichst umfangreiche Zahl an (alternativen) Newswebsites aus Deutschland, Österreich und der deutschsprachigen Schweiz im Zeitraum Oktober 2018 bis August 2019 gesammelt. Die Analysen beziehen sich auf drei Länder (DACH-Raum), da davon ausgegangen wird, dass alternative Nachrichtenmedien nicht nur länderspezifisch ihre Zielgruppen ansprechen, sondern generell im gleichen Sprachraum rezipiert werden - in diesem Fall im deutschsprachigen Raum (Vogler, 2017). Diese Annahme bestätigt sich durch die Analyse der Seitenzugriffe, die meist aus allen drei Ländern stammen (vgl. Kapitel VII.1). Die Länderzugehörigkeit der Seiten wurde erstens durch die URL-Länderkürzel («.de», «.at», «.ch») identifiziert; bei keinem eindeutigen Kürzel (z. B. «.com») wurde zusätzlich das Impressum der Website zur Bestimmung herangezogen. Die Websites wurden induktiv über unterschiedliche Zugänge erhoben: Zunächst sollte eine einfache Suche mittels der Suchmaschine von Google eine erste Grundmenge an Websites hervorbringen. Gearbeitet wurde in diesem Zusammenhang mit den Suchbegriffen «alternative», «kritische», «unabhängige», «freie», «unzensierte» mit dem Zusatz «Nachrichten» oder «Medien» sowie «Deutschland», «Österreich» oder «Schweiz». Die Suchbegriffe stützen sich einerseits auf Definitionen des Begriffs «Alternativmedien», wie im Forschungsstand erörtert, aber auch auf alltagsprachliche Konnotationen mit dieser Nachrichtensorte, die induktiv im Zuge der Recherchen auf den entsprechenden Seiten ergänzt wurden. Ebenso wurden im Oktober 2018 Google Alerts erstellt, die nach ebendiesen Begriffen im Internet suchten, alle Treffer mit diesen Suchbegriffen automatisch auflisteten und per E-Mail retournierten. Über diesen Weg wurden bis August 2019 alle Treffer entsprechend der aufgestellten Kriterien überprüft und in das Sample mitaufgenommen. Kritisch hinterfragt werden kann an dieser Stelle die Suche über Google, zumal für den oder die Nutzer_in nicht eindeutig nachvollziehbar ist, aufgrund welcher Algorithmen die Treffer erscheinen. Dennoch ist anzunehmen, dass jene Seiten, die mit vergleichsweise hohen Reichweiten im Netz öffentlich zirkulieren, durch diesen Weg gefunden wurden - zumal, wie bereits erwähnt, nur jene Seiten für diese Studie von Interesse sind, die öffentlichkeitswirksam im Internet und nicht in geschlossenen Communities agieren. Zusätzlich wurden - um potenzielle Lücken über den Zugang via Google zu minimieren - alle erhobenen Websites im Hinblick auf Verlinkungen auf weitere alternative Nachrichtenwebsites untersucht. Bereits in diesem Schritt wurde deutlich, dass sich viele Websites gegenseitig referenzieren. Insgesamt wurden 178 Websites identifiziert, die den Kriterien entsprechen (vgl. Anhang 1a). Für jede Website wurde zudem unter Verwendung des Websitea- 
nalysetools «Similarweb» (2019) erhoben, wie viele monatliche Seitenzugriffe sie verzeichnet und aus welchen Ländern diese stammen. Weiter mitaufgezeichnet wurden die Präsenz bzw. die Community-Grössen auf den Social-Media-Plattformen Facebook, Twitter und YouTube. Dies mit dem Ziel, ein breites Bild von den Online-Aktivitäten der Nachrichtensites zu erfassen. Der daraus resultierende Datenpool war grundlegend für alle weiteren methodischen Schritte.

\section{VI.2 Qualitative Analyse alternativer Newswebsites}

Nachdem im vorangegangenen ersten methodischen Schritt das Ziel darin bestand, möglichst viele alternative Nachrichtenwebsites zu erheben, um einen breiten Überblick über den derzeitigen Bestand in den drei Untersuchungsländern zu geben, soll im nächsten Schritt ein detaillierter, interpretativer Blick erfolgen. Die Webauftritte der alternativen Nachrichtenmedien werden entsprechend der Forschungsfrage 2 dahingehend untersucht, nach welchen Kriterien sich diese ihrem Selbstverständnis zufolge definieren und kategorisch unterscheiden lassen. Da es sich hierbei um eine offene, explorative Forschungsfrage handelt, mit dem Ziel, eine theoretische Weiterentwicklung dahingehend $\mathrm{zu}$ schaffen, wie sich diese Art von Gegenöffentlichkeiten einordnen lassen, fiel die Wahl auf ein qualitatives Forschungsdesign auf Grundlage der Grounded-Theory-Methodologie. Der Zugang ist phänomenologisch, weshalb das primäre Ziel darin besteht, den subjektiv gemeinten Sinn zu identifizieren, um somit Phänomene erklären zu können. Eine normative Wertung ist in diesem methodischen Schritt zu vermeiden.

Die Methodologie der Grounded Theory (oder: der «gegenstandsbezogenen Theoriebildung») hat eine lange Tradition und im Laufe der letzten Jahrzehnte unterschiedliche Ansätze hervorgebracht. Entwickelt von den amerikanischen Soziologen Barney Glaser und Anselm Strauss (1967) in den 1950er- und 6oer-Jahren besteht das Ziel dieses Forschungsansatzes darin, während des Forschungsprozesses Empirie und Theoriebildung stark miteinander zu verzahnen. Ein Aufstellen von Hypothesen im Vorfeld würde folglich genau dieser Logik widersprechen, da eine höchstmögliche Offenheit bei der Konzeptbildung (im Sinne von Codes und Konstrukten) bewahrt werden soll, die zudem durch zirkuläre Datenerhebungs- und Auswertungsprozesse immer weiterentwickelt werden (Przyborski \& Wohlrab-Sahr, 2014, S. 191ff.). Grundsätzlich wird unter der Grounded Theory eine Theorie verstanden, die induktiv aus der Erforschung des Phänomens selbst entwickelt wird. Sie basiert auf dem Prozess systematischer Datensammlung, Datenauswertung und Theoriebildung. Diese Schritte werden indes nicht nacheinander durchgeführt, sondern stehen im Sinne eines Kreislaufes in wechselseitigem Bezug zueinander (Strauss \& Corbin, 1990, S. 23). Wesentlicher Grund für die Entwicklung der Theorie war das Bestreben der beiden Autoren, die Kluft zwi- 